\title{
Integrated Monitoring of Ecological Conditions in Wetland-Upland Landscapes
}

Landscapes of interwoven wetlands and uplands offer a rich set of ecosystem goods and services, including fresh water, food, fiber, fuel, habitat for biodiversity, genetic reserves, groundwater recharge, pollution control, flood control, sediment retention, biogeochemical cycling, recreation, aesthetics, and climate regulation. Climate and land use can alter the processes that produce these goods and services and reduce the benefits people derive from them.

Managing lands to maximize ecosystem services requires information that distinguishes change caused by local actions from broaderscale shifts in climate, land use, and other forms of global change. Satellite and airborne sensors collect valuable data for this purpose, especially when the data are analyzed along with data collected from ground-based sensors. The U.S. Geological Survey is using remote sensing technology in this way as part of the Terrestrial Wetland Global Change Research Network (TWGCRN; http://www.umesc.usgs.gov/ twgcrn.html). This network of partners and research sites was created to assess effects of climate change interacting with land-use change and other potential stressors along

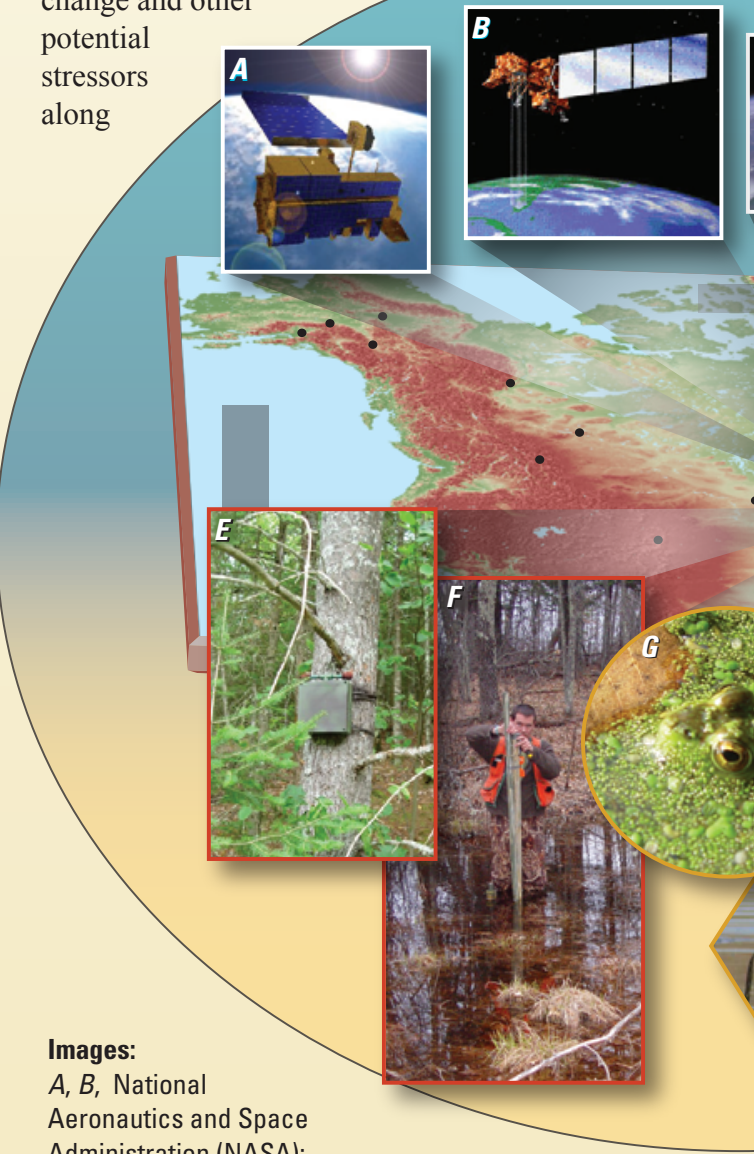

Aeronautics and Space

Administration (NASA);

C, Canada Centre for Remote Sensing;

$D$, Jason Stoker, U.S. Geological Survey;

E, G, I, Mark Roth, U.S. Geological Survey;

$F$, Alisa Gallant, U.S. Geological Survey; and

H, Terry Sohl, U.S. Geological Survey environmental gradients of wetland-upland landscapes in the United States and Canada. TWGCRN partners conduct multiscale assessments of biotic and abiotic conditions using integrated data to (1) establish baselines and measure subsequent changes in landscape conditions, (2) relate landscape changes to altered ecosystem services, and (3) provide resource managers and other stakeholders information vital for mitigating negative impacts of change.

TWGCRN partners from multiple organizations leverage resources across research nodes (fig. 1), each consisting of several individual field sites, to measure change using standard protocols. Partners use sophisticated acoustic recorders to monitor the seasonal presence and temporal dynamics of amphibian and bird populations at these sites relative to other environmental variables. These populations are well-suited as indicators of the effects of climate and land-use change on ecosystem services related to biodiversity and productivity because the species respond to the integrated effects of global change in wetland and terrestrial habitats. Most North American amphibian species reproduce in wetlands but spend a substantial portion of their lives in the surrounding uplands. Many bird species live in close association with wetlands or in a matrix of wetland and upland habitats, not only during the

Figure 1. U.S. and Canadian organizations leverage their resources to measure the impacts of global change in wetlandupland landscapes as part of the Terrestrial Wetland Global Change Research Network (TWGCRN). Data are collected from sensors in the sky and on the ground following standardized protocols to enable networkwide assessments. This figure shows the general locations of research nodes (black dots on map) as of June 2012, with each node representing several wetland sites monitored in situ. Insets $A-D$ are some of the remote sensing systems TWGCRN partners use to characterize landscape and habitat conditions ( $A$, Terra; $B$, Landsat 7; $C$, Radarsat-2; $D$, airborne lidar). Wetlands monitored from the ground are equipped with acoustic sensors $(E)$ to record animal calls, particularly from amphibians and birds (for example, $G$, mink frog; $H$, greater yellowlegs; $I$, wood frog). Measuring other variables in situ (for example, $F$, water-temperature and water-level sensors; $J$, air-temperature sensor) at some sites enables comparing calling activity with weather and hydrologic conditions and, when aggregated, comparing local conditions and responses with those described at broader scales via remotely sensed data. 
breeding season but also while migrating or overwintering in different geographic regions. Thus, amphibian and bird populations respond to changes in their wetland and upland habitats.

Satellite and airborne sensors can provide information over a more extensive area than the local wetland-upland neighborhoods TWGCRN partners monitor with ground-based sensors. These remotely sensed data enable broader, though coarser, assessments across large landscapes (watersheds, regions, continents, and even the globe) that add perspectives on where and when conditions are changing. Integrating local data from ground-based sensors with satellite and airborne sensor data provides critical biotic and abiotic information for understanding key changes and their effects across space and time.

TWGCRN partners use data from several remote sensing systems because each has different capabilities to characterize the environment. Data from the Terra Moderate Resolution Imaging Spectroradiometer (MODIS) sensor have high temporal frequency, but low spatial resolution, and are used to study the timing and vigor of vegetation responses in relation to climate and land use. Data from the Landsat Thematic Mapper (TM) and Enhanced Thematic Mapper Plus (ETM+) sensors have higher spatial resolution, but low temporal frequency, and are used to interpret land-cover patterns (fig. 2A, D) underlying seasonal changes tracked with MODIS data (fig. 2B,F). TWGCRN partners from the Canada Centre for Remote Sensing (http://www.nrcan.gc.ca) are using data from the Radarsat-2 system to map seasonal changes in surface water extent and to develop new approaches to monitor seasonal growth of emergent aquatic vegetation. Data from airborne lidar systems are being used to map where water can collect on the land surface and how the extent of surface water might vary with wetter or drier climate. Integrating this suite of remote sensing information with data from ground-based sensors at research nodes and from weather stations enables TWGCRN partners to measure changing landscape and habitat conditions at different scales relative to the actual responses of amphibian and bird populations in local wetland-upland neighborhoods, invaluable information for resource managers and other stakeholders in a changing world.

Visit http://www.umesc.usgs.gov/twgcrn.htm/ for additional information on the Terrestrial Wetland Global Change Research Network or contact:

Alisa Gallant, TWGCRN Remote Sensing and Geospatial Analysis Lead U.S. Geological Survey

Earth Resources Observation and Science (EROS) Center

Sioux Falls, SD 57198

gallant@usgs.gov

Walt Sadinski, TWGCRN Overall Lead

U.S. Geological Survey Upper Midwest Environmental Sciences Center La Crosse, WI 54603

wsadinski@usgs.gov

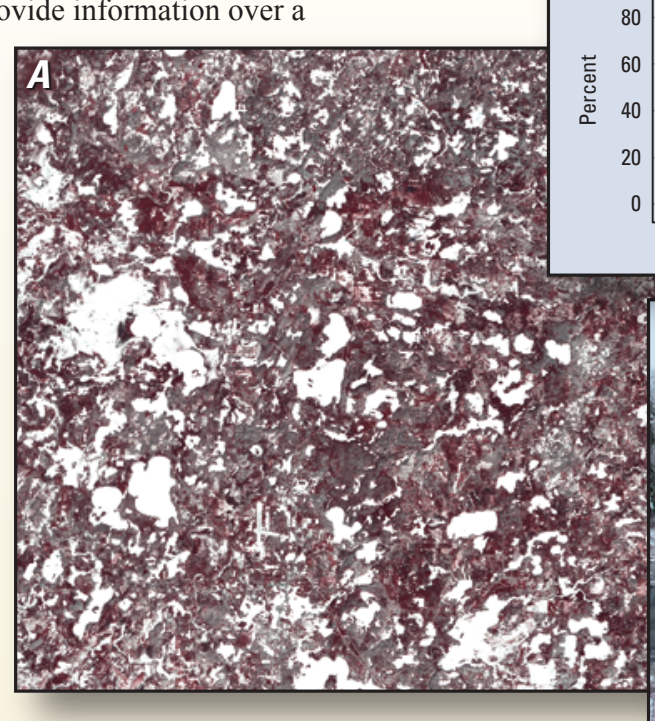

B. Portion of landscape block under snow cover
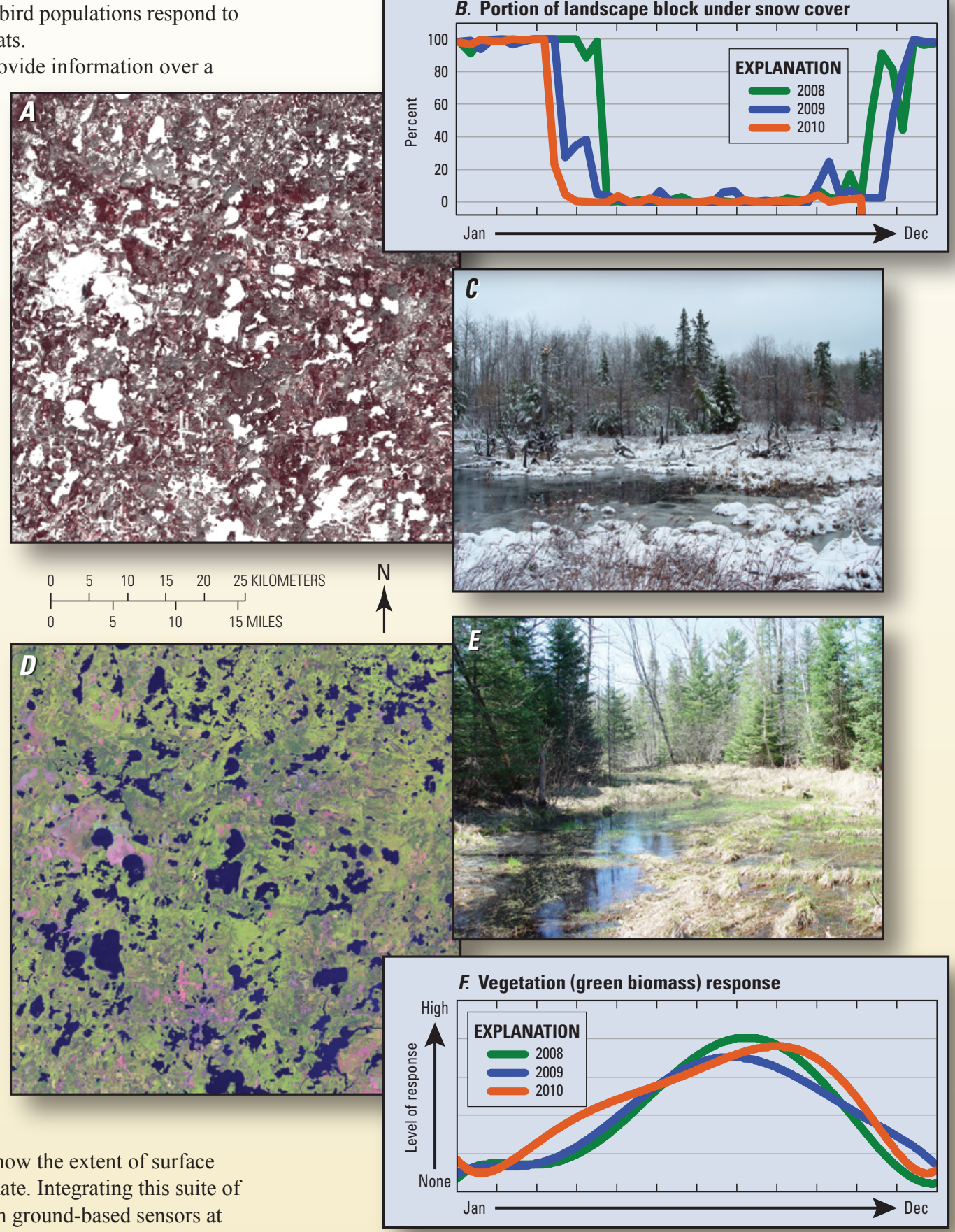

Figure 2. The landscape of the North Temperate Lakes research node (the node highlighted in fig. 1) during late winter/early spring $(A$, Landsat imagery and $C$, ground photo) and early summer $(D$, Landsat imagery and $E$, ground photo). Time-series data from the Terra Moderate Resolution Imaging Spectroradiometer support multiyear comparisons of the timing of snow cover $(B)$ and the green-up and senescence of vegetation $(F$. Annual variations in snow cover, rainfall, and air temperatures result in corresponding differences in the timing and duration $(F, \mathrm{x}$-axis $)$ and vigor $(F, y$-axis $)$ of the vegetation response.

Information on other remote sensing research and data products of USGS EROS is available from:

Customer Services

U.S. Geological Survey Earth Resources Observation

and Science (EROS) Center

Sioux Falls, SD 57198

custserv@usgs.gov 